\title{
Studies of Ultra High Energy Cosmic Rays with the Pierre Auger Observatory
}

\section{H. Lyberis on behalf of the Pierre Auger Collaboration*}

Universidade Federal do Rio de Janeiro, Brazil

E-mail: haris@if.ufrj.br

Aquiring data continuously from 2004, the Pierre Auger Observatory is dedicated to the study of Ultra High Energy Cosmic Rays. In this proceeding, we present the latest Auger results concerning the spectrum, the chemical composition, anisotropy detection and the upper limits on the neutrino and gamma fluxes, constraining the different models of cosmic rays origin.

VIII International Workshop on the Dark Side of the Universe,

June 10-15, 2012

Rio de Janeiro, Brazil

* Speaker. 


\section{Introduction}

A century after the discovery of cosmic rays, their origin is still an open issue. Because the deviation by a magnetic field is inversely proportional to the energy of a charged cosmic ray particle, hopes of directly detecting their sources lie in the detections of the Ultra-High Energy Cosmic Rays (UHECRs). By studying their arrival direction and their nature, the Pierre Auger Observatory is dedicated to the detection of UHECRs using a hybrid detection above a large area. The low flux in this energy range requires measurements of the showers that cosmic ray primaries produce in the atmosphere, namely the extensive air shower (EAS).

There is a general consensus that low energy cosmic rays are of galactic origin, whilst an extra-galactic component is expected to dominate at the highest energies. With their macroscopic energies, UHECRs are thought to be produced in very violent processes in the Universe. In determining their origin, it is important to be able to draw a global picture involving all the different observables that the Pierre Auger Observatory provides.

In this proceeding, we give an overview on the latest results on the different observables related to UHECRs. In the first section we describe the detection methods employed at the Pierre Auger Observatory. The first result, presented in Sec. 3, is the overall spectrum of UHECRs. In the Sec. 4 , we develop the way mass composition is determined by the observatory and present the results. In Sec. 5, we detail the different attempts to detect anisotropies. The last section concentrates on results that constrain different models for Dark Matter (DM).

\section{The Pierre Auger Observatory}

The Pierre Auger Observatory is located near Malarguie, in the Province of Mendoza, Argentina. Determining the part of the sky that is (non-uniformly) seen, the latitude of the site is $35.2^{\circ} \mathrm{S}$ and the mean altitude is $1400 \mathrm{~m}$ above sea level. It has been taking data continuously since January 2004 and its deployment was completed in 2008. Two complementary techniques are used to detect extensive air showers initiated by UHECRs : a surface detector array (SD) and a fluorescence detector (FD).

The SD consists of an array of 1660 water Cherenkov Surface Detectors deployed over a triangular grid of $1.5 \mathrm{~km}$ spacing and covering an area of $3000 \mathrm{~km}^{2}$ [2]. Each station is filled with 12 tons of purified water, and three photomultipliers detect the Cherenkov light produced in the water by secondary particles of the EAS. An SD event is formed when at least 3 neighboring stations are found in spatial and temporal coincidence. Full efficiency, independent of the primary (proton or iron), is achieved above $3 \mathrm{EeV}\left(1 \mathrm{EeV}=10^{18} \mathrm{eV}\right)$. The event direction is determined from a fit to the arrival times of the shower front at the SD. The angular resolution of events having triggered three SD stations is contained within $2.2^{\circ}$ and reaches $1.0^{\circ}$ for events with station multiplicities larger than five [1]. This ground array is overlooked during dark cloudless nights by the FD, composed by 27 fluorescence telescopes, grouped in four sites. The FD observes the longitudinal development of the shower in the atmosphere (i.e. the energy deposit as a function of the atmospheric depth) by detecting the fluorescence light emitted by excited atmospheric nitrogen molecules and Cherenkov light induced by shower particles in air. The FD provides a calorimetric measurement of the primary particle energy, only weakly dependent on theoretical models. An 
event detected by at least one FD telescope and one SD station is called a "hybrid". The combination of the timing information from the FD and the SD provides an accurate determination of the geometry of the air showers. These hybrid events are used to perform an absolute calibration of the corresponding signal recorded by only SD events. Moreover, the use of the hybrid detector allows one to lower the energy threshold for full efficiency to $1 \mathrm{EeV}[2]$.

\section{The UHECRs spectrum}

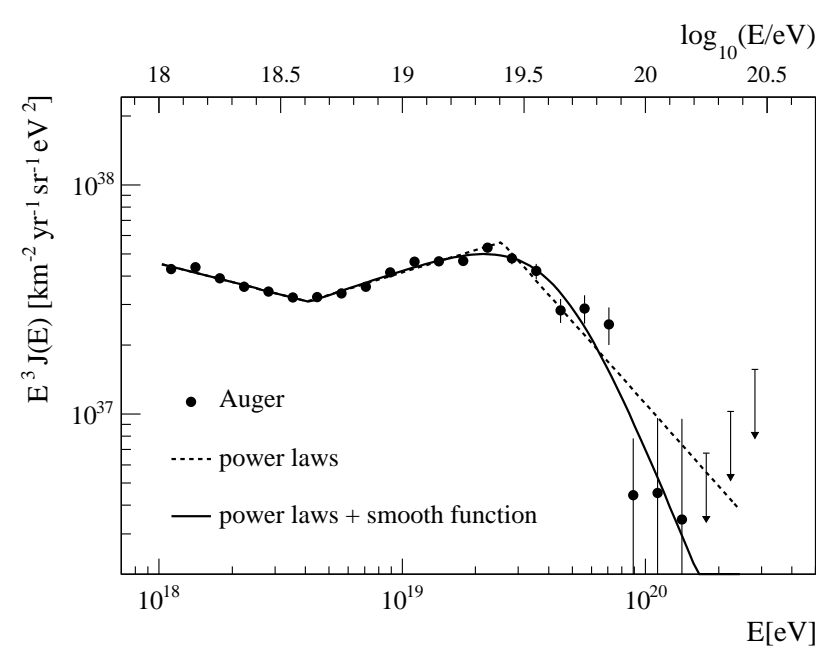

Figure 1: The combined energy spectrum is fitted with two functions (see [4] for further details). Only statistical uncertainties are shown. Both spectra share the same systematic uncertainties in the energy scale of $22 \%$.

An accurate measurement of the cosmic ray flux above $10^{18} \mathrm{eV}$ is a crucial aid for discriminating between different cosmic ray source models, in particular the one describing a transition between galactic and extragalactic cosmic rays. This is related to finding a decrease of the spectrum index, called the ankle. Predicted just after the discovery of the Cosmic Microwave Background (CMB), the so-called GZK cut-off is due to the interaction of extragalactic UHECRs with CMB photons, reducing both the flux and the source distance above $4 \times 10^{19} \mathrm{eV}$. To distinguish between this scenario and features of the injection spectrum at the sources, other observables are required. The two independent measurements of the cosmic ray energy spectrum, with the hybrids and SD events, allows one to draw a combined spectrum ranging from $10^{18} \mathrm{eV}$ to few $10^{20} \mathrm{eV}$ as presented on Fig. 1. Compatible with the GZK prediction, Auger enables a precise measurement of this spectrum.

\section{The chemical composition}

The atmospheric depth at which a shower attains its maximum size, $X_{\max }$, carries information about the mass of the primary particle and the characteristics of hadronic interactions at very high energy. For a given shower, $X_{\max }$ is determined by the depth of the first interaction of the primary in the atmosphere, plus the further depth that it takes the cascade to develop. Extrapolating available 

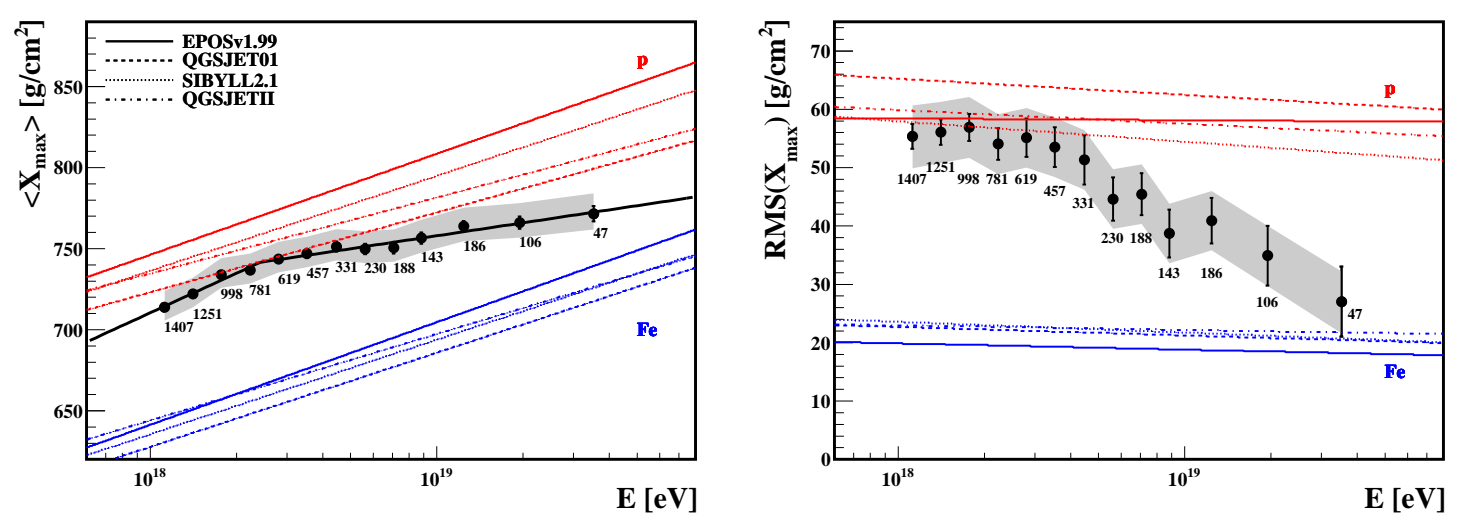

Figure 2: $X_{\max }$ and $\operatorname{RMS}\left(X_{\max }\right)$ distribution using 13 bins of $\Delta \log E=0.1$ below $10^{19} \mathrm{eV}$ and $\Delta \log E=0.2$ above. The "elongation rate" is best described using two slopes.

data to the energies of interest, a qualitative treatment of different nuclear primaries of mass $A$ based on the superposition model indicates that at a given energy $E$, the shower acts as a superposition of $A$ showers of energy $E / A$. Showers of heavier nuclear primaries develop faster that lighter ones. At the same time, fluctuations of the first interaction depth are reduced.

Using data from the FD, measurements of $X_{\max }$ and $\operatorname{RMS}\left(X_{\max }\right)$ as a function of energy are presented on Fig. 2. Energy dependent corrections have been applied to the data to correct for a small bias observed when reconstructing Monte Carlo simulated events, including contributions from uncertainties in the calibration, the atmospheric data, the reconstruction, and the event selection. The dependence on the mass of the primary is qualitatively compatible with the model described here: at a given energy, we expect that for lighter primaries the distribution of $X_{\max }$ will be deeper and broader than for heavier primaries. The small elongation rate at high energies could be interpreted as a change in composition of cosmic rays, from lighter primaries to heavy. At low energy, the shape of the distribution is compatible with a very light or mixed composition, whereas at high energies, the narrow shape would favor a significant fraction of nuclei (CNO or heavier). It is, however, worthwhile noting, that both the mixed composition and the pure iron predictions are at odds with the measured $X_{\max }$. Also, a significant departure from the predictions of available hadronic models would modify this interpretation.

\section{Anisotropies}

Considering UHECRs of extra-galactic origin, the GZK effect implies that above $50-60 \mathrm{EeV}$, the transparent Universe is reduced to an anisotropic box of $\sim 100 \mathrm{Mpc}^{3}$. If the deflections due to extra-galactic magnetic fields are small, one can expect to perform CR astronomy via the detection of point sources in this energy range. Around $1 \mathrm{EeV}$, even if the deflections due to the magnetic fields are expected to isotropize the arrival directions of cosmic, large scale anisotropies are expected to take place. More details on these analyses and complementary studies can be found in [6].

While the first indication of anisotropy in the arrival direction has been given by correlation with astrophysical objects [7], we present here the results of the attempt to evaluate the intrinsic 

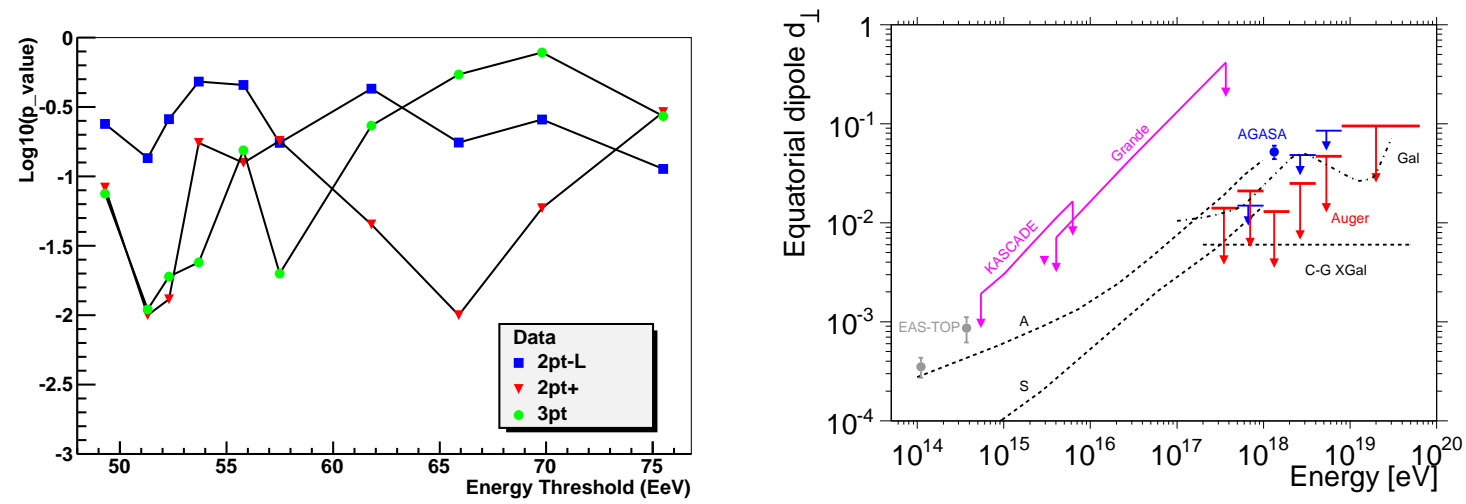

Figure 3: Left panel: Intrinsic anisotropy probabilities in the arrival direction as a function of the energy threshold. Right panel: upper limits on the large scale equatorial dipole amplitude.

anisotropy using methods that do not require any astronomical catalog [8]. The resulting $p-$ values are presented on the left panel of Fig. 3. Being undeflected by magnetic fields, neutrons have a mean decay length of $(9.2 \times(E /[\mathrm{EeV}])) \mathrm{kpc}$ which is comparable to the Earth distance from the Galactic Center. Hence, neutrons could produce a directional excess of cosmic rays in the sky, clustered within the observatory's angular resolution. Two analyses performed at the EeV energy range, (i) a blind search of the exposed sky and (ii) a stacking analysis in the direction of bright gammaray sources detected by the Fermi LAT and H.E.S.S. telescopes, did not reveal any statistically significant excess, allowing one to infer upper limits on the neutron flux.

As a natural signature of the escape of cosmic rays from the galaxy, large scale anisotropies in the distribution of arrival directions might be detected at energies below the ankle. Both the amplitude and the shape of such anisotropies are difficult to predict, as they depend on the model adopted to describe the regular and turbulent components of the galactic magnetic field, the charges of the cosmic rays, and the assumed distribution of sources in space and time. The absence of a detection puts upper limits on the anisotropy dipole amplitude which can be compared to prediction by different models as shown in Fig.3.

\section{Search for neutrinos and photons}

Essentially all models of UHECRs production predict neutrinos and photons as the result of their interactions within the sources themselves, or in their propagation through background radiation fields. Neutrinos and photons are also copiously produced in top-down models proposed as alternatives to explain the production of UHECRs. Some of these models are disfavoured by neutrino and photon upper limits as shown on Fig. 4. With the SD of the Pierre Auger Observatory we can detect and identify UHE neutrinos in the $0.1 \mathrm{EeV}$ range and above. "Earth-skimming" tau neutrinos are expected to be observed through the detection of showers induced by the decay products of an emerging lepton, after the propagation and interaction of a primary neutrino inside the Earth. "Down-going" neutrinos of all flavours can interact in the atmosphere and induce a shower close to the ground. The consistency of the cosmic ray flux suppression beyond $10^{19.6} \mathrm{eV}$ with the predicted GZK cut-off cannot rule out scenarios where this cut-off corresponds to the maximum 

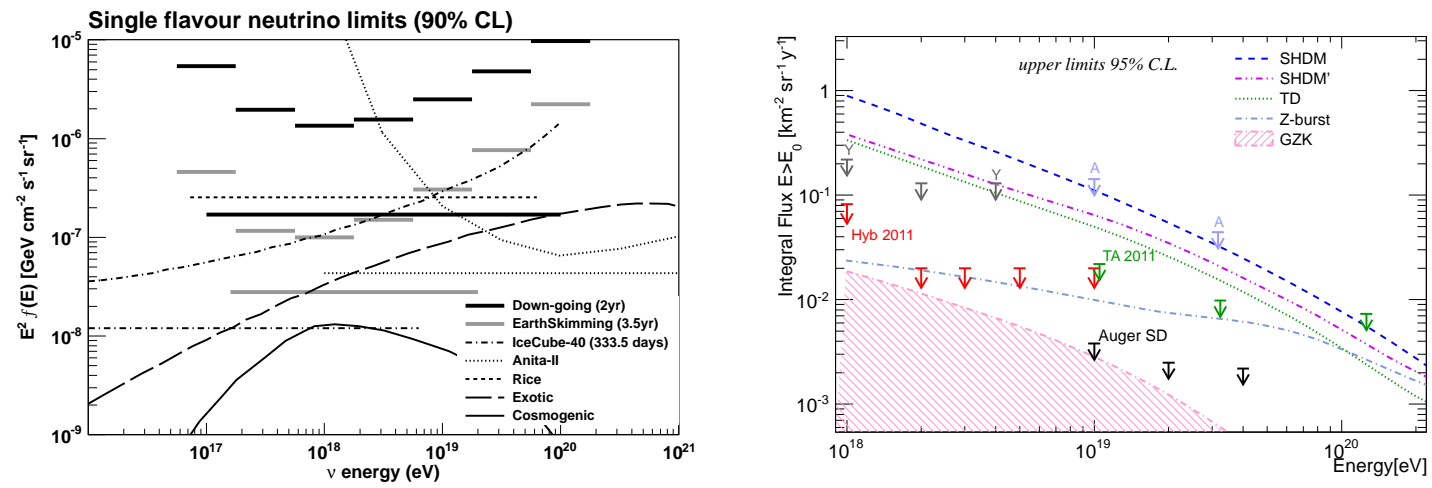

Figure 4: Upper limits on the flux of neutrinos (left panel) and photons (right panel). For the details on the models see reference $[9,10]$.

particle energy reached at the source. The observation of a photon flux compatible with theoretical prediction could provide an independent proof of the GZK process. Two different analyses have been used and the resulting photon upper limits are shown on the right panel of Fig. 4. The first one, based on the SD events, allows one to constrain the flux above $10^{19} \mathrm{eV}$, while a search for $\mathrm{EeV}$ photons was accomplished using hybrid events.

\section{Acknowledgements}

The author would like to thank the whole collaboration for presenting the latest results at this DSU conference and also the Publication Committee for useful comments for this manuscript. This work was supported by CAPES (Grant No. CAPES-PNPD 2940/2011).

\section{References}

[1] C. Bonifazi for the Pierre Auger Collaboration, Nucl. Phys. Proc. Suppl. 190, 20 (2009).

[2] The Pierre Auger Collaboration, Nucl. Instrum. Methods Phys. Res. A 613, 29 (2010).

[3] The Pierre Auger Collaboration, Nucl. Instrum. Methods Phys. Res. A 620, 227 (2010).

[4] M. Settimo for the Pierre Auger Collaboration, Eur. Phys. J. Plus 12787 (2012).

[5] P. Facal San Luis for the Pierre Auger Collaboration, Proceedings of 32th Int. Cosmic Ray Conf. (ICRC 2011) arXiv:1107.4804.

[6] The Pierre Auger Collaboration, Proceedings of 32th Int. Cosmic Ray Conf. (ICRC 2011) arXiv:1107.4805.

[7] The Pierre Auger Collaboration, Astro. Phys. 29, 188 (2008).

[8] The Pierre Auger Collaboration, JCAP 04, 040 (2012).

[9] M. Settimo for the Pierre Auger Collaboration, Proceedings of 32th Int. Cosmic Ray Conf. (ICRC 2011) arXiv:1107.4805.

[10] Y. Guardincerri for the Pierre Auger Collaboration, Proceedings of 32th Int. Cosmic Ray Conf. (ICRC 2011) arXiv:1107.4805. 